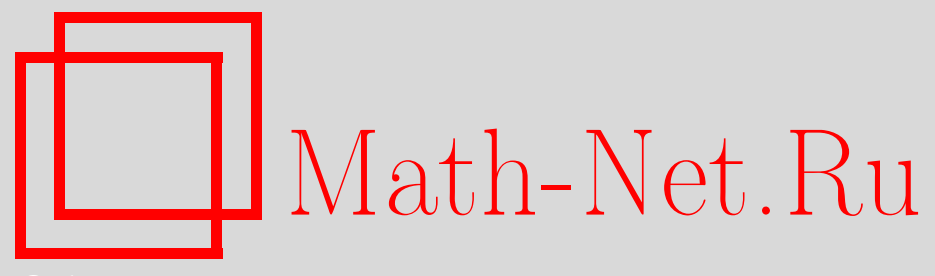

A. M. Zubkov, A. A. Serov, Естественный подход к экспериментальному изучению зависимостей между статистическими критериями, Матем. вопр. криптогр., 2021, том 12, выпуск $1,131-142$

DOI: https://doi.org/10.4213/mvk352

Использование Общероссийского математического портала Math-Net.Ru подразумевает, что вы прочитали и согласны с пользовательским соглашением http://www . mathnet.ru/rus/agreement

Параметры загрузки:

IP : 54.80 .97 .219

26 апреля 2023 г., 13:03:50

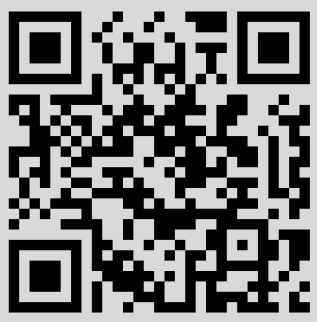


МАТЕМАТИЧЕСКИЕ ВОПРОСЫ КРИПТОГРАФИИ

2021 T. 12 № 1 C. 131-142

УДК 519.254.1+519.233.3

DOI https://doi.org/10.4213/mvk352

\title{
A natural approach to the experimental study of dependence between statistical tests*
}

\author{
A. M. Zubkov, A. A. Serov \\ Steklov Mathematical Institute of Russian Academy of Sciences, Moscow
}

Получено 25.XI.2020

\begin{abstract}
To test the hypothesis on the independence of tests included in the NIST Statistical Test Suite for each of 10000 binary segments we compute the number $\nu$ of tests which reject this segment as not corresponding to the equiprobable Bernoulli sequence hypothesis. If the tests were independent and have the same error probability, then $\nu$ should have the binomial distribution. It appears that collections of 14 and 162 NIST's tests do not satisfy this condition, but 11 tests from 14 may be considered as satisfying it. It is shown also that some sequences obtained by merging seqments of linear recurrent sequences are not rejected by NIST's package.
\end{abstract}

Keywords: independence and uniformity tests, NIST Statistical Test Suite, testing tests independence

\section{Естественный подход $\mathrm{k}$ экспериментальному изучению зависимостей между статистическими критериями}

\section{А. М. Зубков, А. А. Серов}

Математический институт им. В. А. Стеклова Российской академии наук, Москва

Аннотация. Для проверки гипотезы о независимости критериев в пакете NIST для 10000 отрезков двоичных последовательностей вычислялось число $\nu$ критериев, отклонявших гипотезу о том, что это отрезок равновероятной последовательности Бернулли. Для независимых критериев с одной и той же вероятностью ошибки число $\nu$ должно иметь биномиальное распределение. Оказалось, что совокупности из 14 и из 162 критериев пакета NIST не удовлетворяют этому условию, но 11 критериев из 14 удовлетворяют. Показано также, что некоторые последовательности, состоящие из чередующихся отрезков двух линейных рекуррент, не отклоняются критериями пакета NIST.

* The article was submitted by the Organizing Committee of the Symposium CTCrypt'2020.

Citation: Matematicheskie Voprosy Kriptografii, 2021, v. 12, № 1, pp. 131-142 (Russian)

(C) Академия криптографии Российской Федерации, 2021 г. 
Ключевые слова: критерии независимости и равновероятности, статистический пакет NIST, проверка независимости критериев

\section{Introduction}

The problem of testing output binary sequences of Pseudo Random Number Generators (PRNG) is very important for cryptography (as well as for other applications of PRNG). The aim of such testing is to decide whether PRNG generates sequences which may be considered as realizations of equiprobable Bernoulli sequence and therefore may be used in cryptosystems.

Denote by $H_{0}$ the statistical hypothesis that elements of binary sequence are independent and takes values 0 and 1 with probabilities $\frac{1}{2}$. Under this hypothesis all $2^{n}$ binary segments of length $n$ have the same probability $2^{-n}$. So, from probabilistic viewpoint there is no reason to consider some $n$-bit segments as better corresponding to the hypothesis $H_{0}$ than other segments.

In fact each test for the hypothesis $H_{0}$ realizes checking whether there exists some another (not very complicated) hypothesis $H^{*}$ such that the probability of appearance of tested segments under hypothesis $H^{*}$ is essentially larger than under $H_{0}$. Usually the type of $H^{*}$ does not stated explicitly: application of test consists in computation of some statistics (function of tested segments) and hypothesis $H_{0}$ is rejected if the value of this statistics falls into predefined "critical" set having too small probability under $H_{0}$.

Different tests use different statistics and different "critical" sets. Usually testing of segments of binary sequences is based on some well-known batteries of tests, for example, NIST [1], TestU01 [2] etc. Usually all (or many) tests of the battery are applied to each tested segment of sequence.

Let $V_{n}$ be the set of all $2^{n}$ binary segments of length $n$. If the number of tests used to check $H_{0}$ is too large, then almost each segment from $V_{n}$ may be rejected by some tests. If the number of tests used is bounded and $n$ is not small, then large part of segments from $V_{n}$ will not be rejected and this part may contain segments having not very complex structure. (We do not discuss here the approach based on the complexity theory.) For example, in [6] we have constructed simple combinations of pairs of binary linear recurrent sequences which were accepted by all tests of the NIST statistical package with high probability (see below sequences of type MixL).

Nevertheless, despite the shortcomings of statistical testing procedures 
such testing of output sequences generated by PRNG's is necessary: without testing bad PRNG's may be used in cryptosystems and destroy their safety properties.

When a battery of tests is used, some segments may be rejected by several tests. Does it mean that such segments are very bad and that the PRNG producing such segments should be considered as non-admissible? Or this effect may be a consequence of dependencies between tests?

In recent years we have investigated these questions by means of different methods. Some recent results on dependencies between tests included into NIST Statistical Test Suite [1] are presented in this note.

\section{Short review of NIST Statistical Test Suite}

In the complete version of NIST Statistical Test Suite the decision on the acceptance or rejection of hypothesis on the randomness and uniformity of tested sequence is based on the results of 15 tests. The values of statistics of each test are transformed into $p$-values; the whole number of $p$-values equals 188 (148 $p$-values are generated by Non-overlapping Template Matching Test, 8 -values by Random Excursions Test, $18 p$-values by Random Excursions Variant Test, each of Serial and Cumulative Sums Tests generate $2 p$-values). For the study of dependencies between tests we have not considered the Non-overlapping Template Matching Test and two tests connected with Random Excursions (the reasons will be explained at the end of next section). So, we have studied 12 tests generating $14 p$-values, see Table 1.

In [1, Section 4.4] some notes were made on the results of the study on the dependencies between tests included in NIST Statistical Test Suite:

a) by the Kolmogorov-Smirnov test the empirical distributions of $p$ values were compared with the uniform distribution on $[0,1]$,

b) factor analysis of $p$-values was conducted for 161 types of statistics generated by different tests,

c) for pseudorandom sequences of length $10^{6}$ generated by the Blum Blum - Shub generator the principal component analysis was conducted for the set of vectors $z_{j}=\left(z_{1 j}, \ldots, z_{161 j}\right), 1 \leqslant j \leqslant m$, where $z_{i j}=\Phi^{-1}\left(p_{i j}\right)$, $p_{i j}$ is a $p$-value for statistics of $i$-th test computed for $m$-sequence, and $\Phi$ is the distribution function of the standard normal distribution,

d) correlation matrix for vectors $z_{j}, 1 \leqslant j \leqslant m$, was computed. 
Table 1: List of NIST Statistical Tests considered

\begin{tabular}{|c|c|}
\hline No & Name of Test and character of statistics used \\
\hline 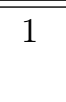 & $\begin{array}{l}\text { Frequency (normed difference between frequencies } \\
\text { of ones and zeros in the whole segment of the sequence) }\end{array}$ \\
\hline 2 & $\begin{array}{c}\text { Block Frequency (relative frequences of ones in adjacent } \\
\text { nonintersecting 128-bit blocks of the segment of the sequence) }\end{array}$ \\
\hline 3 & $\begin{array}{l}\text { Runs (the total number of 1-runs and 0-runs } \\
\text { in the whole segment of the sequence) }\end{array}$ \\
\hline 4 & $\begin{array}{c}\text { Longest Run (maximal lengths of } 1 \text {-runs in adjacent } \\
\text { nonintersecting } 10^{4} \text {-bit blocks of the segment of the sequence) }\end{array}$ \\
\hline 5 & $\begin{array}{c}\text { Binary Matrix Rank (ranks of binary } 32 x 32 \text {-matrices formed from adjacent } \\
\text { nonintersecting 1024-bit blocks of segments of the sequence) }\end{array}$ \\
\hline 6 & $\begin{array}{l}\text { Discrete Fourier Transform (the number of coefficients of discrete Fourier } \\
\text { transform of the } n \text {-bit segment exceeding } h=\sqrt{n \log \frac{1}{0.05}} \text { in absolute value) }\end{array}$ \\
\hline 7 & $\begin{array}{l}\text { Overlapping Template Matching (numbers of 9-bit 1-series in } \\
\text { 1032-bit blocks adjacent intersecting blocks of segment of sequence) }\end{array}$ \\
\hline 8 & $\begin{array}{l}\text { Universal (Maurer's "Universal statistical test") ( } \operatorname{sum} \text { of } \log _{2} \\
\text { of distances between equal nonintersecting 7-bit blocks of } 2^{20} \text {-bit segment) }\end{array}$ \\
\hline 9 & $\begin{array}{l}\text { Linear Complexity (lengths of shortest linear shift registers generating } \\
\text { adjacent nonintersecting 500-bit blocks of the segment of the sequence) }\end{array}$ \\
\hline 10 & $\begin{array}{l}\text { Serial (frequencies of intersecting 16-, } 15 \text { - and 14-bit blocks } \\
\text { in the whole segment of the sequence, results in } 2 \text { statistics) }\end{array}$ \\
\hline 11 & $\begin{array}{l}\text { Approximate Entropy (frequencies of intersecting } \\
\text { 10- and 11-bit blocks in the whole segment of the sequence) }\end{array}$ \\
\hline 12 & $\begin{array}{l}\text { Cumulative Sums (maximal deviation from } 0 \text { for } \pm 1 \text { walk constructed } \\
\text { by a segment of binary sequence, results in } 2 \text { statistics }\end{array}$ \\
\hline
\end{tabular}

The results of these investigations were formulated in [1, section 4.4] as follows:

1) there is no large redundancy among the tests,

2) the degree of duplication among the tests seems to be very small.

Along with this summary the authors of [1] note that there exist dependencies between some tests.

The existence of dependencies between tests of the NIST package were studied in [3]. The author had applied all tests of the NIST package (except Random Excursions and Random Excursions Variant tests) to each of $m$ segments consisting of $n$ bits each, obtaining $162 p$-values for each 
segment. Further for each segment he had computed arithmetic mean of these $p$-values and had compared the histogram corresponding to these $m$ arithmetic means with the normal density approximating the mean of 162 independent random variables having uniform distribution on the interval $(0,1)$. It appears that the histogram is wider than normal density; this is interpreted as the existence of positive correlations between $p$-values.

Note. In [1] and [3] the study of dependencies was concentrated on the global distributions of $p$-values. But decision made by each test depends on the closeness of $p$-value to 0 , so it seems more natural to measure the dependence between tests in terms of left tail dependence between their statistics or $p$-values, or simply between test decisions. In our paper we use the last approach, namely, for each of $m$ tested segments we compute the number of tests rejecting it and compare frequencies of these numbers in the sample of $n$ segments with probabilities of binomial disribution $\operatorname{Bin}(m, q)$, where $q$ is the rejection probability for the segment of equiprobable Bernoulli sequence. This binomial distribution corresponds the case when decisions of tests are independent.

For some simple tests the characteristics of dependence between them may be computed exactly and studied theoretically. For example, in $[5$, theorems $10.3 .3,10.3 .4]$ it was shown that if $s_{0}=0, s_{1}, \ldots, s_{n}$ is the walk on $\mathbb{Z}$ with steps \pm 1 , then the number of walks with $s_{n}=2 w-n$ and $z_{n}=\max _{0 \leqslant k \leqslant n}\left|s_{k}\right| \leqslant u$ is equal to

$$
L(w, n-w, u)=\sum_{k \in \mathbb{Z}}\left(C_{n}^{w-2 k(u+1)}-C_{n}^{w-(2 k-1)(u+1)}\right) .
$$

Analogous combinatorial formula may be found in [4] as a problem to Chapter 3. Since $s_{n}$ and $z_{n}$ correspond the statistics of the Frequency and Cumulative Sums tests, by means of (1) it is possible to compute the correlations and joint distributions of these statistics. It was found that for symmetrical random walk the correlation of $\left|s_{n}\right|$ and $z_{n}$ is approximately 0.85 (depending on $n$ ), and

$$
\mathbf{P}\left\{\left|s_{n}\right|>a, z_{n}>b\right\} \approx \min \left\{\mathbf{P}\left\{\left|s_{n}\right|>a\right\}, \mathbf{P}\left\{z_{n}>b\right\}\right\}
$$

if $\mathbf{P}\left\{\left|s_{n}\right|>a\right\}$ and $\mathbf{P}\left\{z_{n}>b\right\}$ are smaller than 0.01 .

Our experiments had shown that correlations between statistics of Frequency and Cumulative Sums tests are larger than 0.8 . 
In [1] it is recommended to discontinue the Random Excursions and Random Excursions Variant tests if the trajectory of walk with steps \pm 1 constructed by the tested binary segment have smaller than 500 returns to 0 . In [4, Ch.III, $\S 7$, Theorem 4$]$ it is proved that for simple symmetrical random walk $r$-th return to 0 becomes at the moment $n$ with the probability

$$
\varphi_{r, n-r}=\frac{r}{(n-r) 2^{n-r}} C_{n-r}^{n / 2}=r \sqrt{\frac{2}{\pi n^{3}}} e^{-r^{2} / n}\left(1+O\left(\frac{r}{n}+\frac{r^{3}}{n^{2}}\right)\right),
$$

if $n \rightarrow \infty, r=$ const. By means of this formula it is possible to check that for $n=10^{6}$ the probability that the number of returns to 0 will be smaller than 500 is larger than 0.3 , and for $n=10^{7}$ it is larger than 0.1. So, tests based on Random Excursions do not generate any $p$-value for large part of tested segments, and it seems unreasonable to use such tests if the probability of error is chosen to be, for example, 0.01 .

Finally, the Non-overlapping Template Matching Test generates 148 $p$-values and corresponding decisions. "It is highly likely" that among such large family of tests there exist dependent ones. For statistics of frequencies of numbers of tests rejecting the same segment some dependencies between 148 tests may hide dependencies between other tests, so we exclude this test from experiments described below. Dependencies between components of Non-overlapping Template Matching Test will be examined separately.

\section{Description of our experiments}

We have applied 12 tests from Table 1 to pseudorandom sequences of two types: a) sequences generated by a block cipher, b) sequences obtained from pairs of binary linear recurrent sequences.

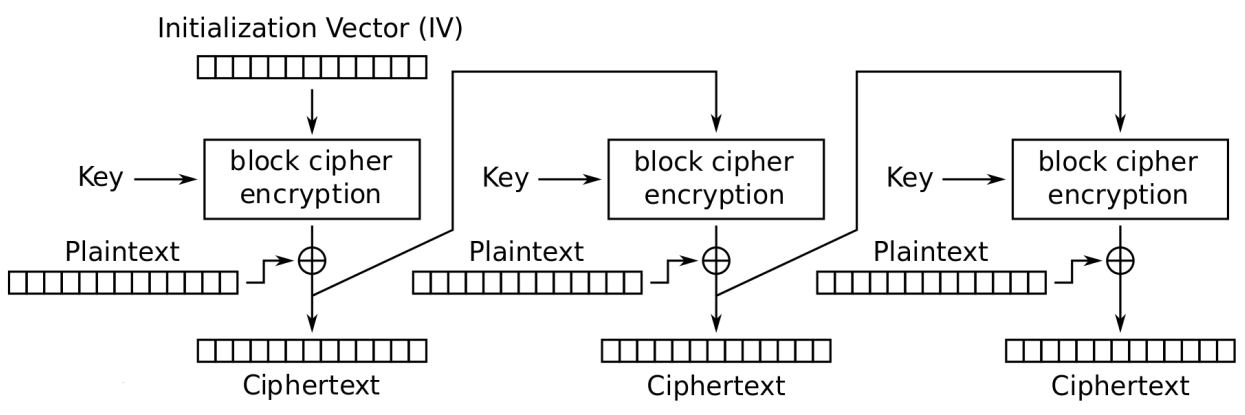

Fig. 1 
The first type sequences were obtained by means of AES block cipher in the CFB (Cipher Feedback Block) mode (see Fig.1) with the zero starting plaintext block, IV=0xfffffffffffffffffffffffffff80, zero key for the sequence denoted by $\mathrm{AES}_{0}$, and key 0x2b7e151628aed2a6abf7158809cf4f3c for the sequence denoted by $\mathrm{AES}_{1}$.

The second type sequences were obtained by quasirandom partitioning two binary linear recurrent sequences into segments and concatenating these segments in alternating manner. We use linear recurrent sequences over GF(2) having maximal periods and characteristic polynomials

$$
\begin{aligned}
f(x) & =x^{43}+x^{27}+x^{22}+x^{5}+1, \\
g(x) & =x^{63}+x+1, \\
h(x) & =x^{33}+x^{26}+x^{22}+x^{21}+x^{3}+x+1, \\
m(x) & =x^{33}+x^{13}+1, \\
q(x) & =x^{63}+x^{60}+x^{22}+x^{18}+x^{8}+x^{5}+1, \\
u(x) & =x^{64}+x^{61}+x^{56}+x^{31}+x^{28}+x^{23}+1 .
\end{aligned}
$$

Initial states of recurrent sequences have only one nonzero element which was the most significant bit.

The recurrent sequences were divided into adjacent segments of pseudorandom lengths according to the following rule:

- the first segment $\left(x_{1}, \ldots, x_{L_{1}^{*}}\right)$ of the first recurrent sequence $\left\{x_{i}\right\}_{i=0}^{2^{n_{1}}-1}$ has length $L_{1}^{*}=64$,

- the first segment $\left(y_{1}, \ldots, y_{L_{2}^{*}}\right)$ of the second recurrent sequence $\left\{y_{i}\right\}_{i=0}^{2^{n_{2}-1}}$ has length

$$
L_{2}^{*}=L_{1}^{*}+2^{6} x_{L_{1}^{*}-6}+2^{5} x_{L_{1}^{*}-5}+2^{4} x_{L_{1}^{*}-4}+2^{3} x_{L_{1}^{*}-3}+2^{2} x_{L_{1}^{*}-2}+2 x_{L_{1}^{*}-1}+x_{L_{1}^{*}},
$$

- the second segment $\left(x_{L_{1}^{*}+1}, x_{L_{1}^{*}+2}, \ldots, x_{L_{1}^{*}+L_{3}^{*}}\right)$ of the first sequence has length

$$
L_{3}^{*}=L_{1}^{*}+2^{6} y_{L_{2}^{*}-6}+2^{5} y_{L_{2}^{*}-5}+2^{4} y_{L_{2}^{*}-4}+2^{3} y_{L_{2}^{*}-3}+2^{2} y_{L_{2}^{*}-2}+2 y_{L_{2}^{*}-1}+y_{L_{2}^{*}},
$$

- and so on.

Tested sequences $\left\{w_{1}, w_{2}, \ldots\right\}$ of the second type have the form

$$
\left\{x_{1}, \ldots, x_{L_{1}^{*}}, y_{1}, \ldots, y_{L_{2}^{*}}, x_{L_{1}^{*}+1}, \ldots, x_{L_{1}^{*}+L_{3}^{*}}, y_{L_{2}^{*}+1}, \ldots\right\} .
$$

Denote tested sequences by corresponding pairs of characteristic polynomials: 


$$
\begin{aligned}
& \operatorname{MixL}_{1}: h(x), m(y), \\
& \operatorname{MixL}_{2}: f(x), g(y), \\
& \operatorname{MixL}_{3}: f(x), q(y), \\
& \operatorname{MixL}_{4}: u(x), q(y) .
\end{aligned}
$$

Each tested sequence was divided into $s=10000$ segments of length $l=2^{20}=1.048 .576$ each (this length corresponds the NIST package recommendations). Each segment was tested by all 12 tests from Table 1.

The application of 12 tests to each segment results in 14 different $p$-values (each of tests 10 and 12 results in two $p$-values). The critical level of $p$-value was chosen as 0.01: if the $p$-value does not exceed 0.01 , then test rejects the hypothesis $H_{0}$ on the randomness and equiprobability of elements of tested segment. For each segment we compute the number of $p$-values not exceeding 0.01 , and for each tested sequence compute frequencies $\eta_{0}, \eta_{1}, \ldots, \eta_{14}$ of these numbers over sample of 10000 volume.

Under the hypothesis on the independence of tests for each segment of "ideal" Bernoulli sequence the number of tests giving $p$-value not exceeding 0.01 should have the binomial distribution with the parameters $(14,0.01)$. Since for each sequence we have considered $s=10000$ segments, then for independent tests

$$
\mathbf{E} \eta_{k}=10000 C_{14}^{k}(0.01)^{k}(0.99)^{14-k}, \quad k=0,1, \ldots, 14 .
$$

To test the hypothesis

$H_{14}:\left(\eta_{0}, \eta_{1}, \ldots, \eta_{14}\right)$ corresponds the sample of 10000 random variables with distribution $\operatorname{Bin}(14,0.01)$

we use the Pearson statistics for $\left(\eta_{0}, \eta_{1}, \eta_{2}+\ldots+\eta_{14}\right)$, joining all frequencies exceeding 1 in one class (to make the mean value of outcomes in each class to be at least 5$)$ :

$$
\text { Pear }=\sum_{k=0}^{1} \frac{\left(\eta_{k}-\mathbf{E} \eta_{k}\right)^{2}}{\mathbf{E} \eta_{k}}+\frac{\left(\sum_{k=2}^{14} \eta_{k}-\sum_{k=2}^{14} \mathbf{E} \eta_{k}\right)^{2}}{\sum_{k=2}^{14} \mathbf{E} \eta_{k}}
$$

Under hypothesis on the independence of tests the distribution of this statistics should be close to the chi-square distribution with 2 degrees of freedom having mean 2 and variance 4 .

Table 2 contains frequencies $\eta_{0}, \eta_{1}, \ldots, \eta_{9}$ for samples of 10000 segments of sequences $\mathrm{AES}_{0}, \mathrm{AES}_{1}, \mathrm{MixL}_{1}, \mathrm{MixL}_{2}, \mathrm{MixL}_{3}, \mathrm{MixL}_{4}$. Last column contains values $\mathbf{E} \eta_{k}$ for the binomial distribution with the parameters 
$(14,0.01)$ (for the case of independent tests). Last row contains values of Pearson statistics (3).

Table 2: Numbers of segments resulting in $k$ (out of 14) p-values smaller than 0.01 and values of Pearson statistics (3) for truncated binomial distribution

\begin{tabular}{|c|c|c|c|c|c|c|c|}
\hline$k$ & $\mathrm{AES}_{0}$ & $\mathrm{AES}_{1}$ & $\mathrm{MixL}_{1}$ & $\mathrm{MixL}_{2}$ & $\mathrm{MixL}_{3}$ & $\mathrm{MixL}_{4}$ & $\mathbf{E} \eta_{k}$ \\
\hline \hline 0 & 8768 & 8826 & 8729 & 5602 & 8816 & 8800 & 8687.46 \\
\hline 1 & 1012 & 992 & 1103 & 1906 & 983 & 1023 & 1228.53 \\
\hline 2 & 125 & 100 & 100 & 978 & 128 & 108 & 80.66 \\
\hline 3 & 83 & 71 & 58 & 734 & 63 & 59 & 3.26 \\
\hline 4 & 12 & 9 & 9 & 496 & 9 & 8 & 0.09 \\
\hline 5 & 0 & 1 & 1 & 158 & 1 & 2 & 0.018 \\
\hline 6 & 0 & 1 & 0 & 59 & 0 & 0 & $2.7 \cdot 10^{-5}$ \\
\hline 7 & 0 & 0 & 0 & 29 & 0 & 0 & $3.2 \cdot 10^{-7}$ \\
\hline 8 & 0 & 0 & 0 & 21 & 0 & 0 & $2.8 \cdot 10^{-9}$ \\
\hline 9 & 0 & 0 & 0 & 17 & 0 & 0 & $1.9 \cdot 10^{-11}$ \\
\hline Total & 10000 & 10000 & 10000 & 10000 & 10000 & 10000 & 10000 \\
\hline Pear & 259 & 162 & 97 & 70487 & 214 & 139 & \\
\hline
\end{tabular}

Table 2 shows that the hypothesis on the independence of tests should be rejected.

Note that frequencies corresponding the sequence $\mathrm{MixL}_{2}$ show that it is rejected by many tests. It may be a consequence of bad mixing properties of linear recurrent sequence with the characteristic polynomial $g(x)$.

More detailed analysis of results of experiments have shown that $p$-values corresponding the Frequency, the Cumulative Sums and the Serial tests are simultaneously smaller than 0.01 with probabilities larger than it should be for independent tests.

If we exclude from the experimental results one of the statistics of the Serial test (number 10 in Table 1) and both statistics of the Cumulative Sums test (number 12 in Table 1), then the remaining tests became more similar to independent ones from the viewpoint of the distribution of the number of tests simultaneously rejecting the same segments. In this case we use equations (2) and (3) with 11 instead of 14. Corresponding results are contained in Table 3 . 
Table 3: Numbers of segments resulting in $k$ (out of 11) $p$-values smaller than 0.01 and values of Pearson statistics (3) for truncated binomial distribution

\begin{tabular}{|c|c|c|c|c|c|c|c|}
\hline$k$ & $\mathrm{AES}_{0}$ & $\mathrm{AES}_{1}$ & $\mathrm{MixL}_{1}$ & $\mathrm{MixL}_{2}$ & $\mathrm{MixL}_{3}$ & $\mathrm{MixL}_{4}$ & $\mathbf{E} \eta_{k}$ \\
\hline \hline 0 & 8878 & 8920 & 8835 & 5650 & 8919 & 8905 & 8953.38 \\
\hline 1 & 1043 & 1016 & 1097 & 2189 & 1012 & 1025 & 994.82 \\
\hline 2 & 78 & 61 & 64 & 1212 & 66 & 66 & 50.24 \\
\hline 3 & 1 & 2 & 4 & 638 & 3 & 4 & 1.52 \\
\hline 4 & 0 & 1 & 0 & 202 & 0 & 0 & 0.03 \\
\hline 5 & 0 & 0 & 0 & 67 & 0 & 0 & $4 \cdot 10^{-4}$ \\
\hline 6 & 0 & 0 & 0 & 25 & 0 & 0 & $4.4 \cdot 10^{-6}$ \\
\hline 7 & 0 & 0 & 0 & 13 & 0 & 0 & $3.2 \cdot 10^{-8}$ \\
\hline 8 & 0 & 0 & 0 & 4 & 0 & 0 & $1.6 \cdot 10^{-10}$ \\
\hline 9 & 0 & 0 & 0 & 0 & 0 & 0 & $5.4 \cdot 10^{-13}$ \\
\hline Total & 10000 & 10000 & 10000 & 10000 & 10000 & 10000 & 10000 \\
\hline Pear & 17.25 & 3.45 & 17.12 & 88539 & 6.14 & 7.57 & \\
\hline
\end{tabular}

Note that after exclusion of three $p$-values the correpondence with hypothesis on independence became better, but nevertheless is far from normal. If $\chi_{2}^{2}$ denotes random variable having the chi-square distribution with 2 degrees of freedom (i.e. exponential distribution with parameter $\frac{1}{2}$ and mean 2), then $\mathbf{P}\left\{\chi_{2}^{2} \geqslant 17.12\right\} \approx 0.0002, \mathbf{P}\left\{\chi_{2}^{2} \geqslant 3.45\right\} \approx 0.178$, $\mathbf{P}\left\{\chi_{2}^{2} \geqslant 6.14\right\} \approx 0.046, \mathbf{P}\left\{\chi_{2}^{2} \geqslant 7.57\right\} \approx 0.023$. Note also that the sequence $\mathrm{AES}_{0}$ was obtained with zero key, sequence $\mathrm{MixL}_{1}$ was constructed from sequences having characteristic polynomials of the same degree 33, sequence $\mathrm{MixL}_{2}$ contains segments of recurrent sequence with three-term characteristic polynomial. So, sequences of these types may have properties which are reflected by some tests. It is interesting that results for sequences $\mathrm{MixL}_{3}, \mathrm{MixL}_{4}$ constructed by merging segments of linear recurrent sequences almost exactly correspond the results for very complex sequences of type $\mathrm{AES}_{1}$.

Similar experiments were performed for the collection of 162 tests of the NIST Statistical Test Suite (Random Excursions and Random Excursions Variant tests were excluded). In this case to test the hypothesis $H_{162}:\left(\eta_{0}, \eta_{1}, \ldots, \eta_{162}\right)$ corresponds the sample of 10000 random variables with distribution $\operatorname{Bin}(162,0.01)$

we use the Pearson statistics for $\left(\eta_{0}, \eta_{1}, \ldots, \eta_{6}, \eta_{7}+\ldots+\eta_{162}\right)$, joining all frequencies exceeding 6 in one class (to make the mean value of outcomes 
in each class to be at least 5):

$$
\text { Pear }=\sum_{k=0}^{6} \frac{\left(\eta_{k}-\mathbf{E} \eta_{k}\right)^{2}}{\mathbf{E} \eta_{k}}+\frac{\left(\sum_{k=7}^{162} \eta_{k}-\sum_{k=7}^{162} \mathbf{E} \eta_{k}\right)^{2}}{\sum_{k=7}^{162} \mathbf{E} \eta_{k}}
$$

Under hypothesis on the independence of tests the distribution of this statistics should be close to the chi-square distribution with 7 degrees of freedom having mean 7 and variance 14 .

Results are given in the next table; it is easy to see that the results do not satisfy the hypothesis on the binomial disribution of the number of tests simultaneously rejecting the segment.

Table 4: Numbers of segments resulting in $k$ (out of 162) $p$-values smaller than 0.01 and values of Pearson statistics (4) for truncated binomial distribution

\begin{tabular}{|c|c|c|c|c|}
\hline$k$ & $\mathrm{AES}_{1}$ & $\mathrm{MixL}_{1}$ & $\mathrm{MixL}_{2}$ & $\mathbf{E} \eta(k)$ \\
\hline \hline 0 & 2176 & 2178 & 1450 & 1962 \\
\hline 1 & 3106 & 3019 & 1401 & 3212 \\
\hline 2 & 2390 & 2481 & 1724 & 2611 \\
\hline 3 & 1323 & 1353 & 1477 & 1407 \\
\hline 4 & 597 & 594 & 1191 & 564 \\
\hline 5 & 275 & 259 & 838 & 180 \\
\hline 6 & 94 & 83 & 544 & 47.7 \\
\hline 7 & 27 & 25 & 361 & 10.7 \\
\hline 8 & 8 & 5 & 237 & 2.1 \\
\hline 9 & 3 & 3 & 159 & 0.36 \\
\hline 10 & 0 & 0 & 94 & 0.06 \\
\hline 11 & 1 & 0 & 75 & 0.008 \\
\hline$\vdots$ & $\vdots$ & $\vdots$ & $\vdots$ & $\vdots$ \\
\hline 141 & 0 & 0 & 1 & $1.03 \cdot 10^{-252}$ \\
\hline Итого & 10000 & 10000 & 10000 & 10000 \\
\hline$S_{\text {Реат }}$ & 197 & 135 & 149600 & \\
\hline
\end{tabular}

\section{Conclusion}

The hypothesis on the independence of tests included in the NIST Statistical Test Suite was rejected on the base of computer experiments by means of statistics of frequencies of numbers of tests simultaneously rejecting the same binary segment. It is shown that from the viewpoint of this statistics 
tests belonging to some subset of NIST tests look more independent than tests of the whole set.

It is shown also that pseudorandom sequences constructed by alternating concatenation of segments of two linear recurrent sequences over GF(2) with non-regularly varying lengths do not rejected by the NIST tests if these recurrent sequences are not too simple.

\section{References}

[1] Rukhin A., Soto J., Nechvatal J., Smid M., Barker E., Leigh S., Levenson M., Vangel M., Banks D., Heckert A., Dray J., Vo S., "A statistical test suite for the validation of random number generators and pseudo random number generators for cryptographic applications", NIST Special Publication 800-22 Revision 1a, 27 April 2010.

[2] L'Ecuyer P., Simard R., “TestU01”, 2013, 214 pp., http://simul.iro.umontreal.ca/testu01/ guideshorttestu01.pdf.

[3] Iwasaki A., "Analysis of NIST SP800-22 focusing on randomness of each sequence", 2017, 8 pp., arXiv: 1710.01441.

[4] Feller W., An Introduction to Probability Theory and its Applications. Vol. 1. 3rd edition, N. Y. e.a.: J.Wiley \& Sons, 1970, 528 pp.

[5] Krattenthaler C., "Lattice path enumeration", Handbook of Enumerative Combinatorics, Boca Raton-London-New York: CRC Press, 2015, 589-678.

[6] Zubkov A. M., Serov A. A., "Testing the NIST Statistical Test Suite on artificial pseudorandom sequences", Matematicheskie Voprosy Kriptografii, 10:2 (2019), 89-96. 